\title{
Nuclear modification factor for light and heavy flavors within pQCD and recent data from the LHC
}

\author{
B.G. Zakharov \\ L.D. Landau Institute for Theoretical Physics, GSP-1, 117940, \\ Kosygina Str. 2, 117334 Moscow, Russia
}

\begin{abstract}
We examine the flavor dependence of the nuclear modification factor $R_{A A}$ in the pQCD calculations at LHC energies. The computations are performed accounting for radiative and collisional parton energy loss with running coupling constant. Our results show that the recent LHC data on the $R_{A A}$ for charged hadrons, $D$-mesons and non-photonic electrons agree reasonably with the pQCD picture of the parton energy loss with the dominating contribution from the radiative mechanism.
\end{abstract}

1. The parton energy loss in the quark-gluon plasma (QGP) is widely believed to be a source for strong suppression of high- $p_{T}$ hadrons in $A A$-collisions (usually called the jet quenching) observed at RHIC and LHC. Understanding the underlying mechanisms of the parton energy loss is of great importance for application of the jet quenching to probing the hot QCD matter produced in $A A$-collisions. In the pQCD picture fast partons lose energy mostly due to induced gluon radiation $[1,2,3,4,5,6]$. The effect of collisional energy loss [7] for the RHIC and LHC conditions is likely to be relatively small [8,9]. Unfortunately, uncertainties in the pQCD-based models of the jet quenching remain large (mostly due to difficulties in modeling multiple gluon emission). For the nuclear modification factor $R_{A A}$ they are perhaps about a factor two. Despite this, it seems relatively safe to assume that predictions for variation of the $R_{A A}$ should be more robust, if the parameters are already adjusted to fit some set of experimental data.

From the point of view of the underlying physics of the jet quenching it is very interesting to compare $R_{A A}$ for light and heavy flavors. It was suggested [10] that for the heavy quarks the dead cone effect should suppress induced gluon emission and give rise to an increase of the $R_{A A}$. However, the observed at RHIC strong suppression of the nonphotonic electrons from the $B / D$-meson decays $[11,12,13]$ seemed to be in contradiction with this picture. It may indicate that for RHIC conditions the dead cone suppression is not very strong or that the radiative mechanism is not the dominating one at all. It stimulated the renewed interest in the collisional energy loss [14]. Although, by adjusting the coupling constant one can obtain a sufficiently strong heavy quark suppression due to the collisional mechanism alone, this scenario does not seem to be realistic (at least for $p_{T} \gtrsim 5-10 \mathrm{GeV}$ ). Calculations of the radiative and collisional energy losses with the 
same $\alpha_{s}$ and the Debye screening mass performed in [8] clearly demonstrate that the collisional loss is relatively small for relativistic partons and unlikely to change significantly the heavy quark energy loss (see also [9]).

In [10] the dead cone suppression was estimated from a qualitative analysis neglecting the quantum finite-size effects. Calculations of the induced gluon emission from heavy quarks in a brick of QGP [15] within the light-cone path integral (LCPI) approach [2], which treats accurately the mass effects, demonstrate that at energy $\sim 10-20 \mathrm{GeV}$ for $c$-quark the induced gluon spectrum is very similar to that for light quarks and $\Delta E_{c} \approx$ $\Delta E_{u, d, s}$, and only for for $b$-quark the gluon emission is suppressed (but not so strongly as predicted by the dead cone model [10]). At high energies ( $\gtrsim 100-200 \mathrm{GeV})$ the radiative energy loss has an anomalous mass dependence with $\Delta E_{b}>\Delta E_{c}>\Delta E_{u, d, s}$ due to the quantum finite-size effects in radiation of hard gluons [15]. In light of these results we can expect that the nuclear modification factor for the heavy quark jets for RHIC and LHC conditions should be qualitatively similar to that for light partons already at $p_{T} \sim 10-20$ $\mathrm{GeV}$. Although accurate simulations and comparison with experiment are needed to reach definite conclusions.

In the present work we examine the flavor dependence of the nuclear modification factor within the LCPI approach [2] and compare our results with the latest LHC data on the $R_{A A}$ for charged hadrons $[16,17], D$-mesons $[18,19]$ and non-photonic electrons [20] in $\mathrm{Pb}+\mathrm{Pb}$ collisions at $\sqrt{s}=2.76 \mathrm{TeV}$. In evaluating the nuclear modification factor, besides the radiative energy loss, we include the collisional one. Both the radiative and collisional contributions are calculated with running $\alpha_{s}$. We account for accurately the fluctuations of the parton path lengths in the QGP. We find that the predicted flavor dependence of the $R_{A A}$ agrees reasonably with the LHC data.

2. We calculate the nuclear modification factor employing the method developed in Ref. [21], to which the interested reader is referred for details. Here we just outline the main aspects of the calculations necessary for understanding of our strategy and interpretation of the results.

For a given impact parameter $b$ the $R_{A A}$ can be written as

$$
R_{A A}(b)=\frac{d N(A+A \rightarrow h+X) / d \mathbf{p}_{T} d y}{T_{A A}(b) d \sigma(N+N \rightarrow h+X) / d \mathbf{p}_{T} d y} .
$$

Here $\mathbf{p}_{T}$ is the particle transverse momentum, $y$ is rapidity (we consider the central region $y=0), T_{A A}(b)=\int d \boldsymbol{\rho} T_{A}(\boldsymbol{\rho}) T_{A}(\boldsymbol{\rho}-\mathbf{b}), T_{A}$ is the nucleus profile function. The differential yield in $A A$-collision can be written in the form

$$
\begin{aligned}
& \frac{d N(A+A \rightarrow h+X)}{d \mathbf{p}_{T} d y}=\int d \boldsymbol{\rho} T_{A}(\boldsymbol{\rho}) T_{A}(\boldsymbol{\rho}-\mathbf{b}) \frac{d \sigma_{m}(N+N \rightarrow h+X)}{d \mathbf{p}_{T} d y} \\
& \frac{d \sigma_{m}(N+N \rightarrow h+X)}{d \mathbf{p}_{T} d y}=\sum_{i} \int_{0}^{1} \frac{d z}{z^{2}} D_{h / i}^{m}(z, Q) \frac{d \sigma(N+N \rightarrow i+X)}{d \mathbf{p}_{T}^{i} d y}
\end{aligned}
$$

Here $\mathbf{p}_{T}^{i}=\mathbf{p}_{T} / z$ is the parton transverse momentum, $d \sigma(N+N \rightarrow i+X) / d \mathbf{p}_{T}^{i} d y$ is the hard cross section, $D_{h / i}^{m}$ is the medium-modified fragmentation function (FF) for transition of a parton $i$ into the observed particle $h$. For the parton virtuality scale $Q$ we take the parton transverse momentum $p_{T}^{i}$. 
We assume that the induced radiation stage occurs after the DGLAP stage which gives the input parton distribution for the induced gluon emission stage. It seems reasonable since for jets with $E \lesssim 100 \mathrm{GeV}$ the typical time scale for the DGLAP stage is relatively small ( $\lesssim 0.3-1 \mathrm{fm}[21]$ ), and in first approximation it is legitimate to neglect interference of the DGLAP and the induced gluon emission stages. Symbolically the medium-modified FF reads

$$
D_{h / i}^{m}(Q) \approx D_{h / j}\left(Q_{0}\right) \otimes D_{j / k}^{i n} \otimes D_{k / i}(Q),
$$

where $\otimes$ denotes $z$-convolution, $D_{k / i}$ is the ordinary DGLAP FF for $i \rightarrow k$ parton transition, $D_{j / k}^{i n}$ is the FF for $j \rightarrow k$ parton transition in the QGP due to induced gluon emission, and $D_{h / j}$ describes parton hadronization outside of the $\mathrm{QGP}^{1}$. In (4) $Q_{0}$ is the scale at which the DGLAP parton showering is stopped. As in [21] we take $Q_{0}=2 \mathrm{GeV}$.

We computed the DGLAP FFs with the help of the PYTHIA event generator [24]. The one gluon induced spectrum, $d P / d x$, was calculated within the LCPI approach [2] employing the method developed in [25]. The $D_{j / k}^{i n}$ has been obtained from $d P / d x$ accounting for multiple gluon emission within Landau's method as in [26]. Note that we include the $q \rightarrow g$ transition as well, which is usually neglected. For the $D_{h / j}\left(Q_{0}\right)$ we use the KKP [27] FFs for light partons, and Peterson FF for heavy quarks (with parameters $\epsilon_{c}=0.06$ and $\left.\epsilon_{b}=0.006\right)$. For the non-photonic electrons we evaluated the FFs $c \rightarrow e$ and $b \rightarrow e$ treating them as the two-step fragmentations $c \rightarrow D \rightarrow e$ and $b \rightarrow B \rightarrow e$. The distributions $B / D \rightarrow e$ were calculated using the CLEO data [28, 29] on the electron spectra in the $B / D$-meson decays. We neglected the $B \rightarrow D \rightarrow e$ process, which gives a negligible contribution [30].

The hard cross sections were calculated using the LO pQCD formula with the CTEQ6 [31] parton distribution functions. To simulate the higher order effects we take for the virtuality scale in $\alpha_{s}$ the value $c Q$ with $c=0.265$ as in the PYTHIA event generator [24]. This prescription allows us to reproduce well the $p_{T}$-dependence of the spectra in $p p$-collisions ${ }^{2}$. In calculating the $R_{A A}$ we account for the nuclear modification of the parton densities (which leads to some small deviation of $R_{A A}$ from unity even without parton energy loss) with the help of the EKS98 correction [32].

As in [21] we take $m_{q}=300$ and $m_{g}=400 \mathrm{MeV}$ for the light quark and gluon quasiparticle masses supported by the analysis of the lattice data [33]. For the heavy quarks we take $m_{c}=1.2 \mathrm{GeV}$ and $m_{b}=4.75 \mathrm{GeV}$. We use the Debye mass obtained in the lattice calculations [34] that give the ratio $\mu_{D} / T$ slowly decreasing with $T\left(\mu_{D} / T \approx 3\right.$ at $T \sim 1.5 T_{c}, \mu_{D} / T \approx 2.4$ at $T \sim 4 T_{c}$ ).

We use the running $\alpha_{s}$ frozen at some value $\alpha_{s}^{f r}$ at low momenta (the technical details for incorporating the running $\alpha_{s}$ can be found in [25]). For gluon emission in vacuum a reasonable choice is $\alpha_{s}^{f r} \approx 0.7[35,36]$. The RHIC data on the pion $R_{A A}$ in $A u+A u$ collisions at $\sqrt{s}=200 \mathrm{GeV}$ support $\alpha_{s}^{f r} \sim 0.5-0.6$ [21]. But the analysis [37] of the first

\footnotetext{
${ }^{1}$ The approximation (4) ignores creation in the QGP of the anomalous jet color states, which may be important for the baryon $R_{A A}$ [22] at not very high $p_{T}$ and the jet structure in the soft region [23]. But it should be reasonable for evaluating the $R_{A A}$ for charged hadrons, which is dominated by the charged pions, and the $R_{A A}$ for heavy flavors.

${ }^{2}$ Although we use the LO formula for the heavy quark cross sections, the $p_{T}$-dependences (and the $c / b$ ratio) of our cross sections agree well with the more sophisticated FONLL calculations [30] (the normalization of the cross sections is unimportant for the $R_{A A}$ at all).
} 
LHC data on the $R_{A A}$ for charged hadrons in $P b+P b$ collisions at $\sqrt{s}=2.76 \mathrm{TeV}$ obtained by ALICE [38] shows that they agree better with $\alpha_{s}^{f r} \sim 0.4-0.5$. The calculations with a fixed coupling constant $[39,40]$ also indicate that it can be smaller at LHC energies. The reduction of $\alpha_{s}^{f r}$ from RHIC to LHC is probably a manifestation of the thermal suppression of $\alpha_{s}$ due to the growth of the initial temperature of the QGP at LHC. We will see that the new data from CMS [16] and ALICE [17] also support $\alpha_{s}^{f r} \sim 0.4-0.5$.

We view the collisional energy loss as a perturbation [21], and account for its effect simply by redefining the initial QGP temperature in calculating the radiative $\mathrm{FF}$ according to the condition

$$
\Delta E_{\text {rad }}\left(T_{0}^{\prime}\right)=\Delta E_{\text {rad }}\left(T_{0}\right)+\Delta E_{\text {col }}\left(T_{0}\right),
$$

where $\Delta E_{\text {rad/col }}$ is the radiative/collisional energy loss, $T_{0}$ is the real initial temperature of the QGP, and $T_{0}^{\prime}$ is the renormalized temperature. We solve (5) in linear approximation in $T_{0}^{\prime 3}-T_{0}^{3}$, which gives $T_{0}^{\prime 3}=T_{0}^{3}+\Delta E_{\text {col }}\left(T_{0}\right) /\left[d E_{\text {rad }}\left(T_{0}\right) / d T_{0}^{3}\right]$. It was done for each parton trajectory in the QGP (separately for quarks and gluons). The collisional energy loss has been evaluated in the Bjorken method [7] with an accurate treatment of kinematics of the binary collisions (the details can be found in [8]).

3. We perform the computations for Bjorken 1+1D longitudinal expansion of the QGP [41], which gives $T_{0}^{3} \tau_{0}=T^{3} \tau$. We take $\tau_{0}=0.5 \mathrm{fm}$. For simplicity we neglect variation of the initial temperature $T_{0}$ in the transverse directions. We evaluated $T_{0}$ using the data on the charged hadron multiplicity pseudorapidity density $d N_{c h} / d \eta[42,43]$ and the entropy/multiplicity ratio $d S / d y / d N_{c h} / d \eta \approx 7.67$ obtained in [44]. It gives $T_{0} \approx 420$ $\mathrm{MeV}$ for central $\mathrm{Pb}+\mathrm{Pb}$ collisions at $\sqrt{s}=2.76 \mathrm{TeV}$. For each jet we calculate accurately the fast parton path length in the QGP, $L$. To take into account the fact that at times about $1-2$ units of the nucleus radius the QGP should cool quickly due to transverse expansion [41], we impose the condition $L<L_{\max }$. We performed the computations for $L_{\max }=8$. The bigger value $L_{\max }=10 \mathrm{fm}$ gives almost the same.

In Fig. 1 we compare the theoretical $R_{A A}$ for charged hadrons obtained for $\alpha_{s}^{f r}=0.5$ and 0.4 to the data from CMS [16] and ALICE [17] for 0-5\% central $P b+P b$ collisions at $\sqrt{s}=2.76 \mathrm{TeV}$. The results are presented for the radiative mechanism alone and with the collisional energy loss. We show our results for $p_{T} \gtrsim 5 \mathrm{GeV}$ since at smaller momenta our perturbative treatment is hardly applicable. Fig. 1 shows that the collisional mechanism suppresses $R_{A A}$ by $\sim 20 \%$ at $p_{T} \sim 10 \mathrm{GeV}$, and $\sim 10 \%$ at $p_{T} \sim 100 \mathrm{GeV}$. One sees that the teoretical $R_{A A}$ (for radiative plus collisional energy loss) for the window $\alpha_{s}^{f r} \sim 0.4-0.5$ agrees reasonably with the experimental data. The agreement is somewhat better for $\alpha_{s}^{f r}=0.4$.

In Fig. 2 we compare our results with the ALICE data $[18,19]$ on the $R_{A A}$ for $D$ mesons in $\mathrm{Pb}+\mathrm{Pb}$ collisions at $\sqrt{s}=2.76 \mathrm{TeV}$ for $0-20 \%$ and $0-7.5 \%$ centrality bins. Fig. 2 shows the results for the $c \rightarrow D$ fragmentation. We found that the effect of the $b$-quark (due to $b \rightarrow B \rightarrow D$ vacuum fragmentation) increases the $R_{A A}$ only by about $2 \%$. From Fig. 2 we can conlude that the same window in $\alpha_{s}^{f r}$ as for light hadrons allows to obtaind a fairly reasonable description of the $D$-meson data as well.

In Fig. 3 we compare our calculations of the $R_{A A}$ for non-photonic electrons with the recent ALICE measurement [20]. We show the contibution from the charm and bottom quarks separately and the total electron $R_{A A}$. Note that for the bottom quark 
our treatment of the collisional mechanism as a pertubation to the radiative one, with the help of (5), loses accuracy at $p_{T} \lesssim 5-6 \mathrm{GeV}$. In this region the collisional correction becomes too large for the predictions to be robust. It happens since the $R_{A A}$ becomes sensitive to the low energy region where for the bottom quark $\Delta E_{c o l} \gtrsim \Delta E_{\text {rad }}$. Evidently, in this regime the radiative and collisional mechanisms must be treated on an even footing. Unfortunately, this problem remains unsolved. For the charm quark this complication does not arise since accross the whole energy range the collisional energy loss remains relatively small [8]. Fig. 3 shows that at $p_{T} \gtrsim 6-7 \mathrm{GeV}$ our results agree with the data fairly well. Note that for the RHIC conditions our results also agree reasonably with the data. For the $0-5 \%$ central $A u+A u$ collisions at $\sqrt{s}=200 \mathrm{GeV}$ for $\alpha_{s}^{f r} \sim 0.5-0.6$ (what is needed for agreement with the $R_{A A}$ for pions) at $p_{T} \sim 6-8 \mathrm{GeV}$ our calculations give the electron $R_{A A} \sim 0.25-0.35$, which agrees reasonably with the STAR [12] measurement. A detailed discusion of the non-photonic electrons for RHIC and LHC energies will be given in a forthcoming publication.

Thus, our pQCD model with the radiative energy loss combined with relatively small collisional energy loss gives a reasonable description of the latest LHC data on the $R_{A A}$ both for light and heavy flavors at $p_{T} \gtrsim 5 \mathrm{GeV}$.

4. In summary, we have examined the flavor dependence of the nuclear modification factor $R_{A A}$ in the pQCD picture and checked its consistency with that observed at LHC. We show that the LHC data on the $R_{A A}$ for charged hadrons [16, 17] and $D$-mesons $[18,19]$ in central $\mathrm{Pb}+\mathrm{Pb}$ collisions at $\sqrt{s}=2.76 \mathrm{TeV}$ can be reasonably described in the pQCD scheme, universal for light and heavy flavors with relatively small collisional energy loss. We found that the ALICE data [20] on the $R_{A A}$ for non-photonic electrons can be described fairly well in our model as well.

We conclude that the recent LHC data on the $R_{A A}$ for the light and heavy jets give strong support for the validity of the pQCD parton mass dependence of the energy loss with relatively small effect of the collisional mechanism. The collisional mechanism becomes very important only for the bottom quark at momenta $\lesssim 6-8 \mathrm{GeV}$. For accurate pQCD calculations in this region a better understanding of the interplay of the radiative and collisional mechanism is required.

\section{Acknowledgements}

I am grateful to the ALICE Collaboration for providing me with the ALICE data shown in Fig. 1. This work is supported in part by the grant RFBR 12-02-00063-a and the program SS-6501.2010.2.

\section{References}

[1] R. Baier, Y.L. Dokshitzer, A.H. Mueller, S. Peigné, and D. Schiff, Nucl. Phys. B483, 291 (1997); ibid. B484, 265 (1997); R. Baier, Y.L. Dokshitzer, A.H. Mueller, and D. Schiff, Nucl. Phys. B531, 403 (1998).

[2] B.G. Zakharov, JETP Lett. 63, 952 (1996); ibid 65, 615 (1997); 70, 176 (1999); Phys. Atom. Nucl. 61, 838 (1998). 
[3] R. Baier, D. Schiff, and B.G. Zakharov, Ann. Rev. Nucl. Part. 50, 37 (2000) [arXiv:hep-ph/0002198].

[4] U.A. Wiedemann, Nucl. Phys. A690, 731 (2001).

[5] M. Gyulassy, P. Lévai, and I. Vitev, Nucl. Phys. B594, 371 (2001).

[6] P. Arnold, G.D. Moore, and L.G. Yaffe, JHEP 0206, 030 (2002).

[7] J.D. Bjorken, Fermilab preprint 82/59-THY (1982, unpublished).

[8] B.G. Zakharov, JETP Lett. 86, 444 (2007) [arXiv:0708.0816].

[9] G.Y. Qin, J. Ruppert, C. Gale, S. Jeon, G.D. Moore, and M.G. Mustafa, Phys. Rev. Lett. 100, 07230 (2008) [arXiv:0710.0605].

[10] Y.L. Dokshitzer and D.E. Kharzeev, Phys. Lett B519, 199 (2001).

[11] S.S. Adler et al. [PHENIX Collaboration], Phys. Rev.L ett. 96, 032301 (2006).

[12] B.I. Abelev et al. [STAR Collaboration], Phys. Rev. Lett. 98192301 (2007) [arXiv:nucl-ex/0607012], Erratum-ibid. 106 (2011) 159902.

[13] A. Adare et al. [PHENIX Collaboration], Phys. Rev. C84, 044905 (2011) [arXiv:1005.1627 [nucl-ex]].

[14] S. Peigné and A. Peshier, Phys. Rev. D77, 114017 (2008) [arXiv:0802.4364]; A. Meistrenko, A. Peshier, J. Uphoff, and C. Greiner, arXiv:1204.2397 and references therein.

[15] P. Aurenche and B.G. Zakharov, JETP Lett. 90, 237 (2009) [arXiv:0907.1918].

[16] CMS Collaboration, Eur. Phys. J. C72 1945 (2012) [arXiv:1202.2554].

[17] ALICE Collaboration, arXiv:1208.2711.

[18] B. Abelev et al. [ALICE Collaboration], JHEP 1209, 112 (2012) [arXiv:1203.2160].

[19] A. Grelli, for the ALICE Collaboration, contribution to the Quark Matter 2012 Conf., http://qm2012.bnl.gov/default.asp.

[20] S. Sakai, for the ALICE Collaboration, contribution to the Quark Matter 2012 Conf., http://qm2012.bnl.gov/default.asp.

[21] B.G. Zakharov, JETP Lett. 88, 781 (2008) [arXiv:0811.0445].

[22] P. Aurenche and B.G. Zakharov, Eur. Phys.J. C71, 1829 (2011) [arXiv:1109.6819].

[23] A. Beraudo, J.G. Milhano, and U.A. Wiedemann, Phys. Rev. C85, 031901 (2012) [arXiv:1109.5025].

[24] T. Sjostrand, L. Lonnblad, S. Mrenna, and P. Skands, arXiv:hep-ph/0308153. 
[25] B.G. Zakharov, JETP Lett. 80, 617 (2004) [arXiv:hep-ph/0410321].

[26] R. Baier, Yu.L. Dokshitzer, A.H. Mueller, and D. Schiff, JHEP 0109, 033 (2001).

[27] B.A. Kniehl, G. Kramer, and B. Potter, Nucl. Phys. B582, 514 (2000).

[28] A.H. Mahmood et al. [CLEO Collaboration], Phys. Rev. D70, 032003 (2004).

[29] R. Poling, invited talk at 4th Flavor Physics and CP Violation Conference, Vancouver, British Columbia, Canada, 9-12 Apr 2006, arXiv:hep-ex/0606016.

[30] M. Cacciari, P. Nason, and R. Vogt, Phys. Rev. Lett. 95, 122001 (2005).

[31] S. Kretzer, H.L. Lai, F. Olness, and W.K. Tung, Phys. Rev. D69, 114005 (2004).

[32] K.J. Eskola, V.J. Kolhinen, and C.A. Salgado, Eur. Phys. J. C9, 61 (1999).

[33] P. Lévai and U. Heinz, Phys. Rev. C57, 1879 (1998).

[34] O. Kaczmarek and F. Zantow, Phys. Rev. D71, 114510 (2005).

[35] N.N. Nikolaev and B.G. Zakharov, Phys. Lett. B327, 149 (1994).

[36] Yu.L. Dokshitzer, V.A. Khoze, and S.I. Troyan, Phys. Rev. D53, 89 (1996).

[37] B.G. Zakharov, JETP Lett. 93, 683 (2011) [arXiv:1105.2028].

[38] K. Aamodt et al. [ALICE Collaboration], Phys. Lett. B696, 30 (2011).

[39] W.A. Horowitz and M. Gyulassy, Nucl. Phys. A872, 265 (2011) [arXiv:1104.4958].

[40] B. Betz and M. Gyulassy, Phys. Rev. C86, 024903 (2012) [arXiv:1201.0281].

[41] J.D. Bjorken, Phys. Rev. D27, 140 (1983).

[42] S. Chatrchyan et al. [CMS Collaboration], JHEP 1108, 141 (2011) [arXiv:1107.4800].

[43] K. Aamodt et al. [ALICE Collaboration], Phys. Rev. Lett. 106, 032301 (2011).

[44] B. Müller and K. Rajagopal, Eur. Phys. J. C43, 15 (2005). 


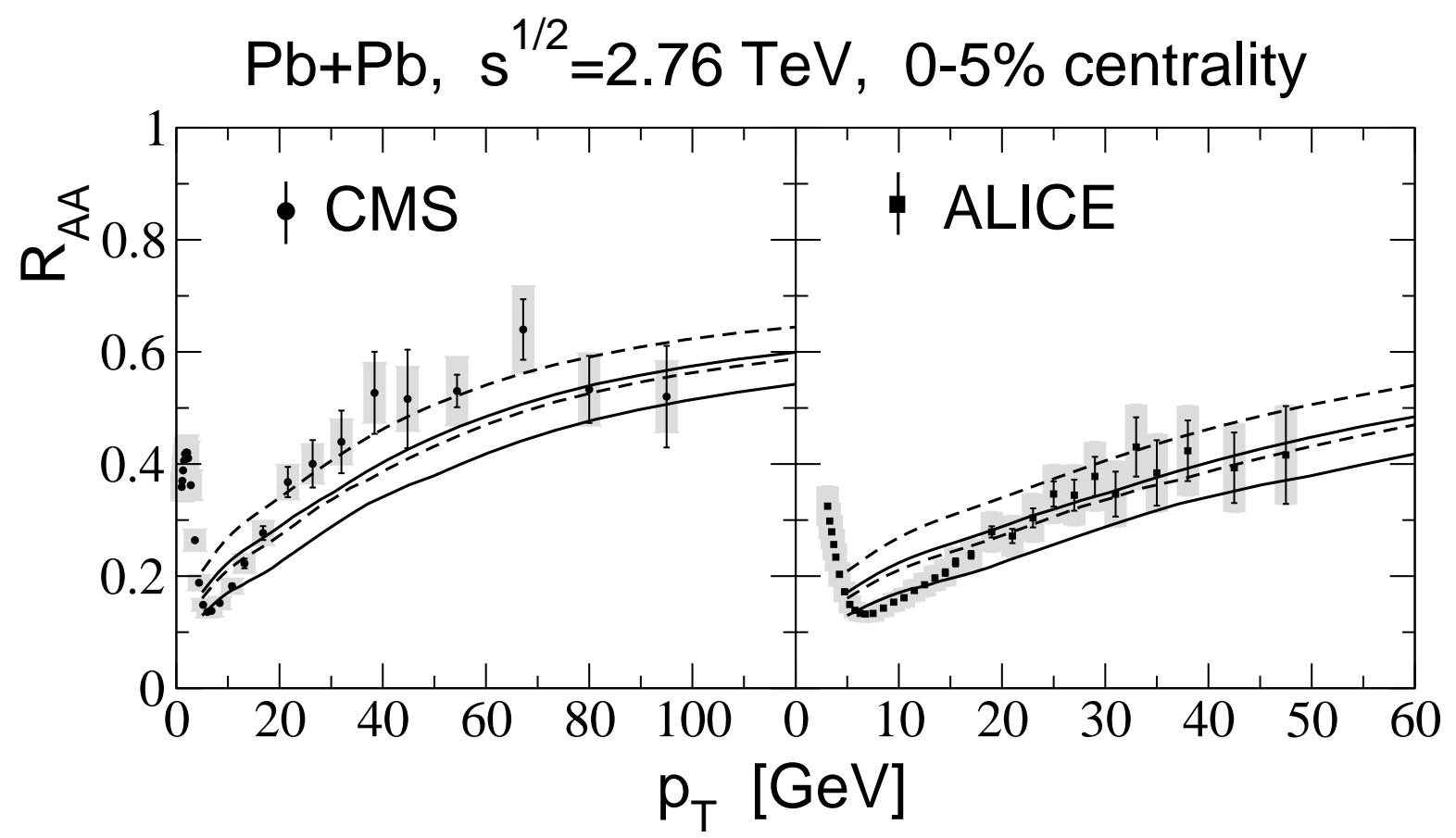

Figure 1: The nuclear modification factor for charged hadrons at $y=0$ for $0-5 \%$ central $P b+P b$ collisions at $\sqrt{s}=2.76 \mathrm{TeV}$ for $\alpha_{s}^{f r}=0.4$ (upper curves) and 0.5 (lower curves). The solid line shows the calculations with the radiative and collisional energy loss, and the dashed line shows the results for the radiative mechanism alone. The experimental points are the data from CMS [16] (circles) and ALICE [17] (squares). Systematic errors are shown as shaded areas. 


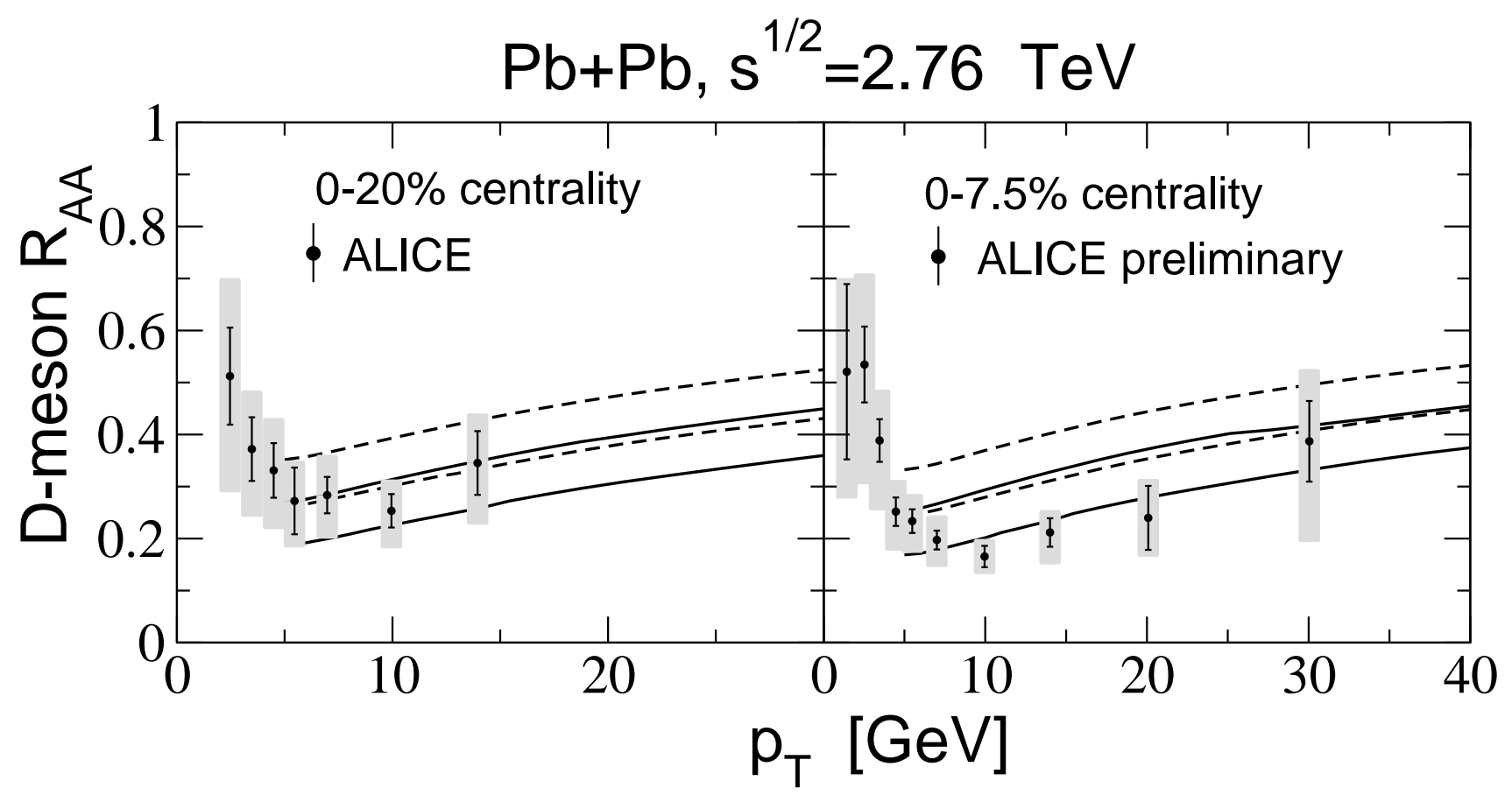

Figure 2: The $D$-meson nuclear modification factor for $0-20 \%$ (left) and $0-7.5 \%$ (right) central $\mathrm{Pb}+\mathrm{Pb}$ collisions at $\sqrt{s}=2.76 \mathrm{TeV}$ for $\alpha_{s}^{f r}=0.4$ (upper curves) and 0.5 (lower curves) at $y=0$. The solid line shows the calculations with the radiative and collisional energy loss, and the dashed line shows the results for the radiative mechanism alone. The experimental points are the ALICE data [18] (left panel), [19] (right panel) for average $D^{0}, D^{+}, D^{*}$. Systematic errors are shown as shaded areas. 


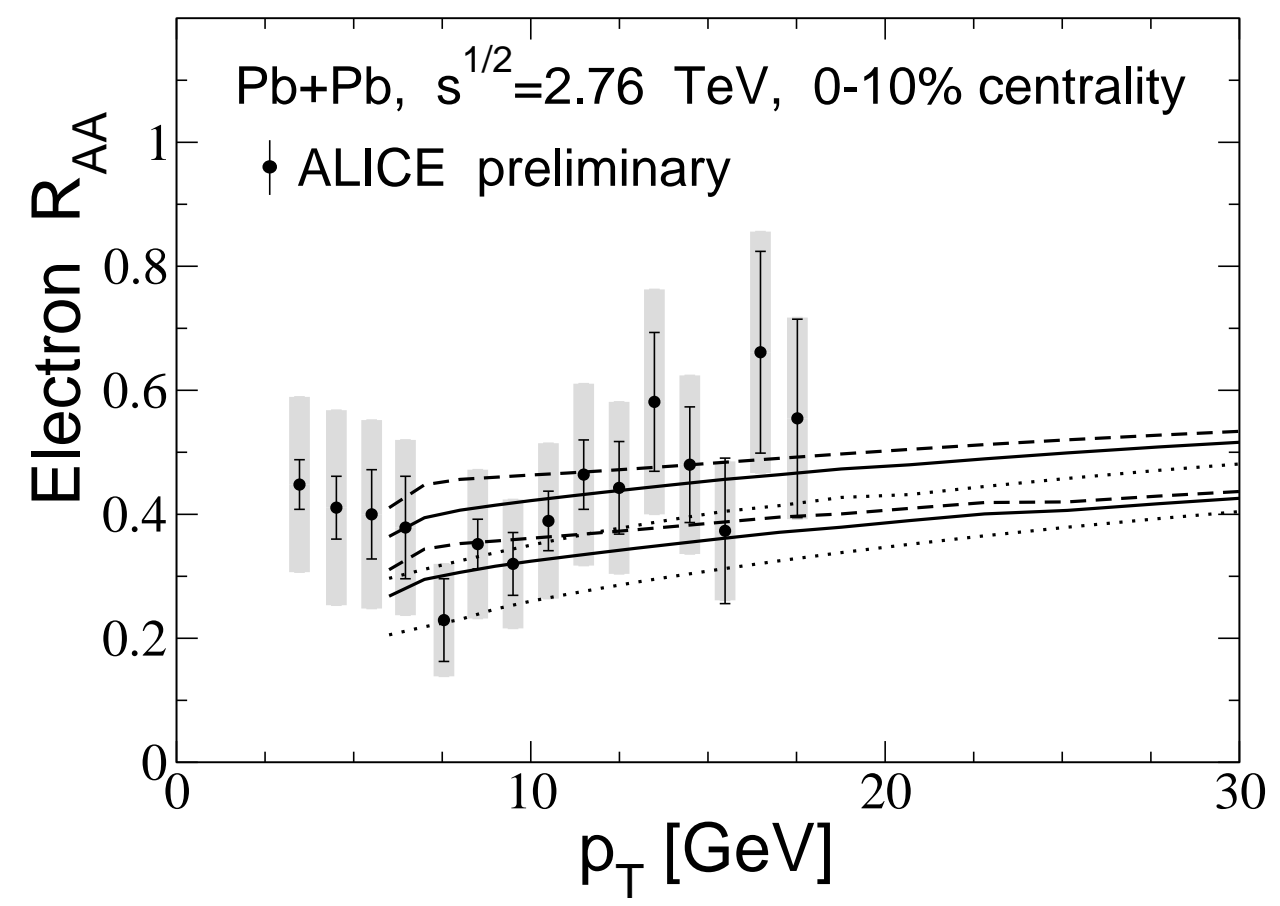

Figure 3: The electron nuclear modification factor for $0-10 \%$ central $\mathrm{Pb}+\mathrm{Pb}$ collisions at $\sqrt{s}=2.76 \mathrm{TeV}$ for $\alpha_{s}^{f r}=0.4$ (upper curves) and 0.5 (lower curves) at $y=0$. The solid line shows the total $R_{A A}$, the dotted and dashed lines show the $R_{A A}$ for charm and bottom contributions, respectively. The experimental points are the preliminary ALICE data [20]. Systematic errors are shown as shaded areas. 